\title{
Adjuvant electrochemotherapy with bleomycin and cisplatin combination for canine soft tissue sarcomas: A study of 30 cases
}

\author{
Enrico P. Spugnini ${ }^{1, *}$, Bruno Vincenzi ${ }^{2}$, Bruno Amadio ${ }^{3}$ and Alfonso Baldi ${ }^{4,5}$ \\ ${ }^{1}$ Biopulse srl, Naples, Italy \\ ${ }^{2}$ Medical Oncology Department, University Campus Bio-Medico of Rome, Rome, Italy \\ ${ }^{3}$ SAFU service, Regina Elena Cancer Institute, Rome, Italy \\ ${ }^{4}$ Department of Environmental, Biological and Pharmaceutical Sciences and Technologies Campania University \\ "Luigi Vanvitelli", Caserta, Italy \\ ${ }^{5}$ Institute of Biosciences and BioResources, CNR, Naples, Italy
}

\begin{abstract}
Electrochemotherapy (ECT) couples the administration of anticancer drugs with the delivery of electric pulses that increase the drug uptake through the cell membranes, thus resulting in an improved efficacy. This study has evaluated the tolerability and efficacy of the combination of systemic bleomycin and local cisplatin as ECT agents for incompletely excised canine soft tissue sarcoma (STS). Thirty dogs with incompletely excised STSs were enrolled. The dogs received intravenous $20 \mathrm{mg} / \mathrm{m}^{2}$ bleomycin, and the tumor bed and margins were infiltrated with cisplatin at the dose of $0.5 \mathrm{mg} / \mathrm{cm}^{2}$. Then, trains of permeabilizing biphasic electric pulses were applied under sedation. More precisely, 5 min after the injection of the chemotherapy agents, sequences of eight biphasic pulses lasting $50+50 \mu$ sec each, were delivered in bursts of $1,300 \mathrm{~V} / \mathrm{cm}$ using caliper electrodes. A second session was performed $2 \mathrm{wk}$ later. The treatment was well tolerated and side effects were minimal. Twenty-six dogs had no evidence of recurrence at the time of manuscript writing; four had recurrence and one of the four recurring dogs died of lung metastases. Median estimated disease free was $857 \mathrm{~d}$. Perivascular wall tumors response was compared to that of the other STSs, but the difference in outcome was not significant. ECT using combination of bleomycin and cisplatin appears to be effective in the treatment of incompletely resected STSs in dogs. This therapeutic approach could be a useful addition to the current options in consideration of its low cost, limited toxicity, and ease of administration.
\end{abstract}

Keywords: Bleomycin, Cisplatin, Dog, Electrochemotherapy, Sarcoma.

\section{Introduction}

Soft tissue sarcomas (STSs) are an assorted pool of solid tumors categorized together due to their clinical and histopathological features (Ehrhart, 2005; Bray et al., 2014). STSs represent approximately $15 \%$ of all canine skin cancers, with a yearly incidence of 35 cases per 100,000 patients at risk (Liptak and Forrest, 2013). These tumors usually present as soft or firm masses with poorly defined margins and frequent infiltration of the neighboring tissues (Dernell et al., 1998). Therefore, conservative surgery is unable to achieve a complete eradication of the tumor, thus frequently resulting in recurrence (Mayer and LaRue, 2005). Some prognostic factors for recurrence have been identified, such as incompleteness of resection versus radical excision (Chase et al., 2009), or tumor grade (Bray et al., 2014). Hematological dissemination is a common event, being observed in up to $20 \%$ of cases (Liptak and Forrest, 2013). Response to chemotherapy and radiation therapy is mostly limited to neoplasms smaller than $5 \mathrm{~cm}$ in diameter (Liptak and Forrest, 2013). The most commonly adopted strategies aimed at increasing local control involved the combination of surgery with adjuvant therapies, such as radiation therapy (Forrest et al., 2000; McKnight et al., 2000), sometimes associated with hyperthermia (Thrall et al., 2005), adjuvant chemotherapy (Selting et al., 2005), and electrochemotherapy (ECT) (Spugnini et al., 2007a, 2008a, 2011). ECT is an anticancer therapy that couples the administration of chemotherapy agents with the delivery of trains of permeabilizing electric pulses having an appropriate waveform (Spugnini et al., 2014, 2016, 2017). These pulses induce transient perturbation and rearrangement of the cell membrane, resulting in increased cross-flow of material through the cytoplasmic membrane, including low permeant molecules, such as bleomycin and cisplatin (Silve and Mir, 2011). Direct electrical destruction of cells is a negligible effect, caused by the inability of some cells to sequester calcium within the sarcoplasmic reticulum (Silve and Mir, 2011). The improved chemotherapy uptake by cancer cells induces tumor apoptotic death (Silve and Mir, 2011). Local cisplatin has been used to increase local control with good results, especially for grade I and II STS (Bergman et al., 2016). Nevertheless, since the first ECT studies, it has been shown that 
electroporation greatly increases the efficacy of cisplatin, compared to controls treated with cisplatin alone (Sersa et al., 1998; Tozon et al., 2001). Several studies have been investigating ECT for the treatment of STS in pets showing that it was a useful addition to surgery (Spugnini et al., 2007b, 2008a, 2011). In this study, we evaluated the tolerability and effectiveness of a combined ECT protocol involving local and systemic administration of chemotherapy agents (cisplatin and bleomycin, respectively) in dogs carrying incompletely excised STS.

\section{Materials and Methods}

Thirty-three dogs with histopathologically confirmed, incompletely excised sarcomas were referred for adjuvant ECT. A patient present with a large grade II peripheral nerve sheath tumor of the left foreleg was shown in Figure 1A. Patients, staged according to the World Health Organization (WHO) grading system, were treated if they fulfilled the following criteria: i) accessibility of the neoplasm location; ii) absence of distant metastases; iii) absence of other life-threatening diseases such as cardiac disease and renal failure; and iv) absence of bone involvement. Patients were staged through the measurement of the surgical field, histopathological exam of tumor, regional lymph node fine-needle aspiration biopsy (when feasible), complete blood cell count, chemistry profile, urinalysis, chest radiographs, and abdominal ultrasonography. Canine patients received two sessions of ECT 2 weeks apart, starting at the time of suture removal (10-14 d after surgery). Dogs were pre-medicated with acepromazine, butorphanol, and medetomidine, followed by propofol to effect. Bleomycin was intravenously injected as a bolus at the dose of $20 \mathrm{mg} / \mathrm{m}^{2}$. Moreover, aiming at improving local control, the tumor bed (a surface of two centimeters deep under the surgical scar, or in case of leg location, as deep as feasible and two additional centimeters of margins at each side), was infiltrated with cisplatin at the dose of $0.5 \mathrm{mg} / \mathrm{cm}^{2}$. Five minutes after the chemotherapy injections, sequences of eight biphasic pulses lasting $50+50 \mu \mathrm{sec}$ each, were delivered in bursts of $1,300 \mathrm{~V} / \mathrm{cm}$ using caliper electrodes until the treatment coverage was achieved as elsewhere described (Spugnini et al., 2011). An application of plate electrodes on the tumor bed of a patient is shown in Figure 1B. Pulses were generated using an electroporator certified for veterinary use (Onkodisruptor $\left.{ }^{\circledR}\right)$ (. 1C). Contact between the patients and the electrodes was optimized using an electroconductive gel. The treatment was repeated after 2 weeks. During the ECT sessions, the patients were monitored using a cardiac monitor and pulse oximeter.

\section{Results}

Thirty-three patients were referred for adjuvant treatment over a period of $4 \mathrm{yr}$. Three dogs were lost to follow-up, leaving 30 patients for statistical
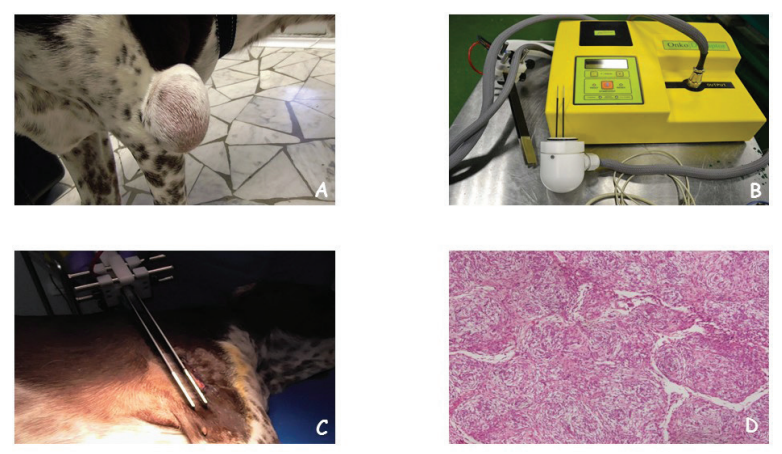

Fig. 1. (A) A dog with a large grade II peripheral nerve sheath tumor of the left foreleg at the presentation. (B) Permeabilizing electric pulses are administered using plate electrodes. (C) The electroporator used for the therapies. (D) The histopathology of the tumor showing the typical alternating Antoni A and B areas (Hematoxylin and Eosin; original magnification $\times 10$ ).

analysis. Specifically, the remaining dogs belonged to the following breeds: 20 mixed breed dogs, one Rottweiler, one Beagle, one dobermann, one bulldog, one hunting dog, one German shepherd, two dachshunds, and two Abruzzese shepherd. Age varied from 3 to $14 \mathrm{yr}$ with a median of $10 \mathrm{yr}$. Tumor histotypes were the following: nine perivascular wall tumors, six peripheral nerve sheath tumors, six fibrosarcomas, three chondrosarcomas, two hemangiosarcomas, two liposarcomas, and two undifferentiated sarcomas. The histological features of a peripheral nerve sheath tumor are depicted in Figure 1D. Patients characteristics including histological grade and site of the tumour are summarized in Table 1. The treatment was well tolerated and side effects were confined to local inflammation in three dogs that were controlled through the administration of an antiinflammatory drug (firocoxib as per manufacturer's instructions). Systemic side effects were not identified. In terms of response, twenty-six dogs $(86.6 \%)$ had no evidence of recurrence at different times (Table 1), four had recurrence, and one of the four recurring dogs (affected by hemangiosarcoma) died of lung metastases (Table 1). Two of the twentysix responders died of unrelated causes at different times. Median disease free was not reached at the time of writing: the Kaplan-Meier estimated disease free of $857 \mathrm{~d}$ for all the canine population (Fig. 2A). In the attempt to identify prognostic factors, a comparison between the larger cohort of tumors (perivascular wall tumors) versus all the other tumor histotypes was performed since they are also considered the best responders to therapy (Stefanello et al., 2008; Avallone et al., 2014). Again, median disease free was not reached and the calculated estimated disease free values were: $1,162 \mathrm{~d}$ versus 992 , respectively ( $p$ $=0.222)$ (Fig. 2B). 
Table 1. Patients' characteristics and outcome.

\begin{tabular}{|c|c|c|c|c|c|}
\hline Age (Years) & Tumor site & T stage & Histotype & Disease free* & Outcome \\
\hline $81 / 2$ & Left foreleg & T3 & PWT Grade II & 483 & NED \\
\hline 13 & Right foreleg & $\mathrm{T} 3$ & U-SA & 93 & Recurrence, euthanasia \\
\hline $21 / 2$ & Right hindleg & $\mathrm{T} 2$ & HSA & 1053 & NED \\
\hline 9 & Head & $\mathrm{T} 3$ & PNST Grade II & 1505 & NED \\
\hline 8 & Thorax & $\mathrm{T} 3$ & FSA Grade II & 1249 & NED \\
\hline 9 & Right foreleg & $\mathrm{T} 2$ & PWT Grade I & 1298 & NED \\
\hline 10 & Left foreleg & $\mathrm{T} 2$ & PWT Grade II & 1235 & NED \\
\hline 3 & Right foreleg & $\mathrm{T} 3$ & U-SA & 366 & Recurrence, amputation \\
\hline 4 & Abdomen & $\mathrm{T} 3$ & CSA & 856 & Dead, renal failure \\
\hline 10 & Lumbar & $\mathrm{T} 3$ & PWT Grade II & 840 & NED \\
\hline $101 / 2$ & Head & $\mathrm{T} 2$ & HSA & 366 & Recurrence,metastasis \\
\hline 10 & Right hindleg & $\mathrm{T} 3$ & LipoSA & 443 & Recurrence, amputation \\
\hline 8 & Left hindleg & $\mathrm{T} 3$ & PWT Grade II & 692 & NED \\
\hline 6 & Right foreleg & $\mathrm{T} 2$ & PWT Grade II & 660 & NED \\
\hline 14 & Thorax & $\mathrm{T} 3$ & PNST Grade II & 413 & Dead, cardiac disease \\
\hline 4 & Flank & $\mathrm{T} 3$ & LipoSA & 604 & NED \\
\hline 6 & Flank & $\mathrm{T} 3$ & FSA Grade II & 574 & NED \\
\hline $131 / 2$ & Head & $\mathrm{T} 2$ & $\mathrm{CSA}$ & 513 & NED \\
\hline 10 & Left hindleg & $\mathrm{T} 2$ & PWT Grade II & 370 & NED \\
\hline 13 & Perineum & $\mathrm{T} 2$ & FSA Grade II & 370 & NED \\
\hline 7 & Perineum & $\mathrm{T} 3$ & FSA Grade II & 338 & NED \\
\hline 8 & Flank & $\mathrm{T} 3$ & CSA & 286 & NED \\
\hline 10 & Right foreleg & $\mathrm{T} 3$ & PNST Grade III & 283 & NED \\
\hline 11 & Left foreleg & $\mathrm{T} 2$ & PNST Grade II & 269 & NED \\
\hline 10 & Abdomen & $\mathrm{T} 3$ & PNST Grade II & 584 & NED \\
\hline 3 & Flank & $\mathrm{T} 3$ & FSA Grade II & 212 & NED \\
\hline 6 & Right foreleg & $\mathrm{T} 2$ & PWT Grade II & 209 & NED \\
\hline 5 & Left foreleg & $\mathrm{T} 2$ & FSA Grade II & 209 & NED \\
\hline 8 & Right foreleg & $\mathrm{T} 2$ & PWT Grade II & 205 & NED \\
\hline 8 & Flank & $\mathrm{T} 3$ & PNST Grade II & 149 & NED \\
\hline
\end{tabular}

(PWT): perivascular wall tumor; (U-SA): undifferentiated sarcoma; (HSA): hemangiosarcoma; (PNST): peripheral nerve sheat tumor; (FSA): fibrosarcoma; (CSA): chondrosarcoma; (LipoSA): liposarcoma; (NED): no evidence of disease; (*): Days.

\section{Discussion}

Our results provide valuable insight into long-term outcome of dogs with STS treated with combination adjuvant ECT. In STS surgery, clean resection margins remain the gold standard, with several studies claiming increased overall and disease-free survival times with wider surgical margins (Kuntz et al., 1997; Dernell et al., 1998; Ehrhart, 2005). General orientation for canine STSs calls for en-block resection with 2-3 cm of lateral margins, and the inclusion of one fascial plane deep to the tumor bed (Chase et al., 2009; Bray et al., 2014; Hohenhaus et al., 2016). However, this optimal surgical procedure is rarely accomplished with most canine sarcomas still being marginally excised (Kuntz et al., 1997; Dernell et al., 1998; Ehrhart, 2005; Bray et al., 2014). Recent clinical evidence suggests that increasing the extension of surgical excision is not enough to improve the treatment success for all STSs (Bray et al., 2014). Moreover, beside the aggressiveness of surgical technique, other factors play a pivotal role in STSs control, including size, histotype, and histologic grade (McSporran, 2009; Bray et al., 2014). Other management options include a combination of surgery plus radiotherapy and chemotherapy (Forrest et al., 2000; McKnight et al., 2000; Simon et al., 2007; Demetriou et al., 2012). Radiotherapy is currently the elective adjuvant treatment for 

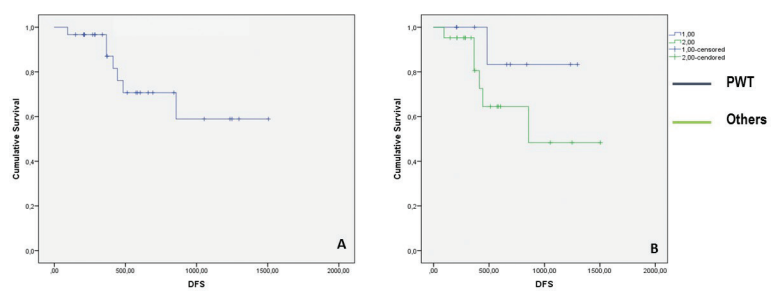

Fig. 2. (A) Kaplan-Meyer curve showing the time to recurrence for 30 dogs with STS treated with adjuvant ECT. (B) Kaplan-Meyer curve showing the time to recurrence for dogs with peripheral wall tumors versus other sarcomas.

incompletely excised solid tumors. However, it has some limitations: 1) availability of a radiation facility, 2) cost of the treatment, and 3) possibility of serious but non-life-threatening local complications such as wound dehiscence, self-trauma, and osteonecrosis of underlying bone (Demetriou et al., 2012). ECT is an anticancer therapy that associates the systemic and/or local administration of chemotherapy agents with the delivery of permeabilizing electric pulses with specific amplitude, duration, and waveforms (Spugnini et al., 2016, 2017). This strategy is helpful to increase the efficacy of lipophobic drugs that often have a narrow therapeutic index, thus minimizing patient toxicoses while maintaining adequate anticancer efficacy (Spugnini et al., 2014). ECT has been described as a useful adjuvant therapy for the treatment of both feline and canine STSs [Reviewed in Spugnini et al. (2016)]. Regarding its application in STSs, ECT is almost exclusively used in an adjuvant modality since these neoplasms show a limited response to ECT alone (Spugnini et al., 2016). Consequently, ECT has been adopted as an adjuvant therapy after surgical excision of STSs in several canine and feline studies (Spugnini et al., 2007b, 2008a, 2011). In these studies, the chemotherapy agent (either bleomycin or cisplatin) was injected in the tumor bed. All these studies evidenced a clear clinical gain in terms of local control. In all these adjuvant studies, side effects were limited to local inflammation and occasional wound dehiscence, with the exception of a previously radiated cat that experienced a radiation recall (Spugnini et al., 2007a, 2008b, 2009, 2011). This is the first report of a combined ECT approach in veterinary medicine with cisplatin and bleomycin. Systemic bleomycin administration increased the likelihoods of drug distribution in the deeper layers of the tumor bed, while the local injection of cisplatin was aimed at increasing ECT efficacy in the superficial layers. Our results show that this combination can improve the control of aggressive STS with limited side effects, even better than our previous published canine protocol. In terms of response, we have $86.6 \%$ of the treated subjects, still in remission at the time of writing compared to $50 \%$ of the previous study (Spugnini et al., 2007a).
Furthermore, the estimated disease free interval in the current study is $859 \mathrm{~d}$ (median time not reached), while it was of $730 \mathrm{~d}$ in the previous study (Spugnini et al., 2007a). Moreover, we observed a greater disease-free interval for perivascular wall tumors compared to other STSs although this phenomenon was not statistically significant. Generally speaking, perivascular wall tumors are less prone to recurrence than other histotypes, especially if they are located in the extremities (Stefanello et al., 2011). In particular, it has been underlined that for those neoplasms, size, grade $(>I)$, and location of the tumor were significant prognostic factors, while status of margins had only a clinically relevant hazard ratio (Stefanello et al., 2011). In our patients cohort, the tumors we treated were mostly grade II or higher and had also a considerable size (T2 or T3) although most of the perivascular wall tumors (PWTs) were located in the extremities. This supports the increased local control obtained with ECT in our patients. Surgery remains the cornerstone for the treatment of STSs, being most of the time able to achieve local control by itself, especially for certain types such as the PWTs (Stefanello et al., 2008, 2011; Bray et al., 2014). In case of incomplete excision for tumors that are likely to recur, adjuvant therapies are advocated, and among them, ECT displays a reasonable ratio between cost and benefits, as also shown by the current literature (Spugnini et al., 2007a, 2011). In the future studies, we plan to increase the number of cases treated with this combination protocol and to expand the tumor histotypes analyzed, in order to confirm the data presented and to identify subgroups that are more responsive. In conclusion, our data suggest that ECT can be considered an additional adjuvant therapy to increase local control of canine STS. We propose to consider it an effective addition to the current anticancer strategies for STS in veterinary medicine.

\section{Acknowledgments}

Partially presented at the ACVIM Forum 2018, June 14-16, Seattle USA. Enrico P. Spugnini and Alfonso Baldi are stockholders of Biopulse S.r.1.

\section{References}

Avallone, G., Boracchi, P., Stefanello, D., Ferrari, R., Rebughini, A. and Roccabianca, P. 2014. Canine perivascular wall tumors: high prognostic impact of site, depth, and completeness of margins. Vet. Pathol. 51, 713-721.

Bergman, N.S., Urie, B.K., Pardo, A.D. and Newman, R.G. 2016. Evaluation of local toxic effects and outcomes for dogs undergoing marginal tumor excision with intralesional cisplatin-impregnated bead placement for treatment of soft tissue sarcomas: 62 cases (2009-2012). J. Am. Vet. Med. Assoc. 248, 1148-1156. 
Bray, J.P., Polton, G.A., McSporran, K.D., Bridges, J. and Whitbread, T.M. 2014. Canine soft tissue sarcoma managed in first opinion practice: outcome in 350 cases. Vet. Surg. 43, 74-82.

Chase, D., Bray, J., Ide, A. and Polton G. 2009. Outcome following removal of canine spindle cell tumours in first opinion practice: 104 cases. J. Small Anim. Pract. 50, 568-574.

Demetriou, J.L., Brearley, M.J., Constantino-Casas, F., Addington, C. and Dobson J. 2012. Intentional marginal excision of canine limb soft tissue sarcomas followed by radiotherapy. J. Small Anim. Pract. 53, 174-181.

Dernell, W.S., Withrow, S.J., Kuntz, C.A. and Powers, B.E. 1998. Principles of treatment for soft tissue sarcoma. Clin. Tech. Small Anim. Pract. 13, 59-64

Ehrhart, N. 2005. Soft-tissue sarcomas in dogs: a review. J. Am. Anim. Hosp. Assoc. 41, 241-246.

Forrest, L.J., Chun, R., Adams, W.M., Cooley, A.J. and Vail DM. 2000. Postoperative radiotherapy for canine soft tissue sarcoma. J. Vet. Intern. Med. 14, 578-582.

Hohenhaus, A.E., Kelsey, J.L., Haddad, J., Barber, L., Palmisano, M., Farrelly, J. and Soucy, A. 2016. Canine cutaneous and subcutaneous soft tissue sarcoma: an evidence-based review of case management. J. Am. Anim. Hosp. Assoc. 52, 7789.

Kuntz, C.A., Dernell, W.S., Powers, B.E., Devitt, C., Straw, R.C. and Withrow, S.J. 1997. Prognostic factors for surgical treatment of soft-tissue sarcomas in dogs: 75 cases (1986-1996). J. Am. Vet. Med. Assoc. 211, 1147-1151.

Liptak, J.M. and Forrest, L.J. 2013. Soft tissue sarcomas. In Small animal clinical oncology, 4th ed. Eds., Withrow, S.J., Vail, D.M. and Page, R.L., Elsevier, St Louis, Missouri, USA, pp: 356-380.

Mayer, M.N. and LaRue, S.M. 2005. Soft tissue sarcomas in dogs. Can. Vet. J. 46, 1048-1052.

McKnight, J.A., Mauldin, G.N., McEntee, M.C., Meleo, K.A. and Patnaik, A.K. 2000. Radiation therapy for incompletely resected soft tissue sarcomas in dogs. J. Am. Vet. Med. Assoc. 217, 205-210.

McSporran, K.D. 2009. Histologic grade predicts recurrence for marginally excised canine subcutaneous soft tissue sarcomas. Vet. Pathol. 46, 928-933.

Selting, K.A., Powers, B.E., Thompson, L.J., Mittleman, E., Tyler, J.V., Lafferty. M.H. and Withrow, S. 2005. Outcome of dogs with high-grade soft tissue sarcomas treated with and without adjuvant doxorubicin chemotherapy: 39 cases (1996-2004). J. Am. Vet Med. Assoc. 227, 1442-1448.

Sersa, G., Stabuc, B., Cemazar, M., Jancar, B., Miklavcic, D. and Rudolf, Z. 1998. Electrochemotherapy with cisplatin: potentiation of local cisplatin antitumour effectiveness by application of electric pulses in cancer patients. Eur. J. Cancer 34, 1213-1218.

Silve, A. and Mir, L.M. 2011. Cell electropermeabilization and cellular uptake of small molecules: the electrochemotherapy concept. In Clinical aspects of electroporation, 1st ed. Eds., Kee, S.T., Gehl, J., Lee, E.W., Springer, New York,NY, USA, pp: 69-82.

Simon, D., Ruslander, D.M., Rassnick, K.M., Wood, C.A., Frimberger, A.E., Cotter, S.M., King, N.W. and Moore, A.S. 2007. Orthovoltage radiation and weekly low dose of doxorubicin for the treatment of incompletely excised soft-tissue sarcomas in 39 dogs. Vet. Rec. 160, 321-326.

Spugnini, E.P., Vincenzi, B., Citro, G., Santini, D., Dotsinsky, I., Mudrov, N., Montesarchio, V., Laieta, M.T., Esposito, V. and Baldi, A. 2007a. Adjuvant electrochemotherapy for the treatment of incompletely excised spontaneous canine sarcomas. In Vivo 21, 819-822.

Spugnini, E.P., Baldi, A., Vincenzi, B., Bongiorni, F., Bellelli, C., Citro, G. and Porrello, A. 2007b. Intraoperative versus postoperative electrochemotherapy in high grade soft tissue sarcomas: a preliminary study in a spontaneous feline model. Cancer Chemother. Pharmacol. 59, 375-381.

Spugnini, E.P., Vincenzi, B., Betti, G., Cordahi, F., Dotsinsky, I., Mudrov, N., Citro, G. and Baldi, A. 2008a. Surgery and electrochemotherapy of a highgrade soft tissue sarcoma in a dog. Vet. Rec. 162, 186-188.

Spugnini, E.P., Dotsinsky, I., Mudrov, N., Citro, G., Caruso, G., Cardelli, P. and Baldi, A. 2008b. Electrochemotherapy-induced radiation recall in a cat. In Vivo 22, 751-753.

Spugnini, E.P., Renaud, S.M., Buglioni, S., Carocci, F., Dragonetti, E., Murace, R., Cardelli, P., Vincenzi, B., Baldi, A. and Citro, G. 2011. Electrochemotherapy with cisplatin enhances local control after surgical ablation of fibrosarcoma in cats: an approach to improve the therapeutic index of highly toxic chemotherapy drugs. J. Transl. Med. 9, 152.

Spugnini, E.P., Melillo, A., Quagliuolo, L., Boccellino, M., Vincenzi, B., Pasquali, P. and Baldi, A. 2014. Definition of novel electrochemotherapy parameters and validation of their in vitro and in vivo effectiveness. J. Cell Physiol. 22, 1177-1181.

Spugnini, E.P., Azzarito, T., Fais, S., Fanciulli, M. and Baldi, A. 2016. Electrochemotherapy as first line cancer treatment: experiences from veterinary medicine in developing novel protocols. Curr. Cancer Drug Targets 16, 43-52.

Spugnini, E.P., Fais, S., Azzarito, T. and Baldi, A. 2017. Novel instruments for the implementation of electrochemotherapy protocols: from bench side to veterinary clinic. J. Cell Physiol. 232, 490-495. 
Stefanello, D., Morello, E., Roccabianca, P., Iussich, S., Nassuato, C., Martano, M., Squassino, C., Avallone, G., Romussi, S. and Buracco, P. 2008. Marginal excision of low-grade spindle cell sarcoma of canine extremities: 35 dogs (1996-2006). Vet. Surg. 37, 461-465.

Stefanello, D., Avallone, G., Ferrari, R., Roccabianca, P. and Boracchi, P. 2011. Canine cutaneous perivascular wall tumors at first presentation: clinical behavior and prognostic factors in 55 cases. J. Vet. Intern. Med. 25, 1398-1405.
Thrall, D.E., LaRue, S.M., Daohai, Y., Samulski, T., Sander, L., Case, B., Rosner, G., Azuma, C., Poulson, J., Pruitt, A.F., Stanley, W., Hauck, M.L., Williams, L., Hess, P. and Dewhirst, M.W. 2005. Thermal dose is related to duration of local control in canine sarcomas treated with thermoradiotherapy. Clin. Cancer Res. 11, 5206-5214.

Tozon, N., Sersa, G. and Cemazar, M. 2001. Electrochemotherapy: potentiation of local antitumour effectiveness of cisplatin in dogs and cats. Anticancer Res. 21, 2483-2488. 